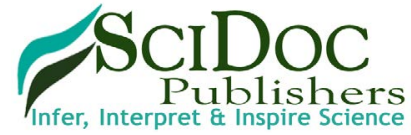

\title{
PAR-2-Mediated Relaxation of Rectum is Impaired Following Bacillary Dysentery in Rat
}

Research Article

Luo $\mathrm{Y}^{1,2}$, Wang $\mathrm{Q}^{3}$, Liu $\mathrm{C}^{1 *}$

${ }^{1}$ Department of Physiology, Shandong University School of Medicine, Jinan, PR China.

${ }^{2}$ Department of Physiology, School of Basic Medical Science, Ningxia Medical University, Yinchuan, PR China.

${ }^{3}$ Key Laboratory of the Qilu University, Shandong University, Jinan, PR China.

\section{Abstract}

Background: PAR-2 activation modifies the intestinal motility and takes part in gastrointestinal motility disturbances under intestinal infection or inflammation, but its role in the regulation of normal rectum motility and its change following bacillary dysentery remains unclear.

Methods: Healthy male wistar rats, weighing 200-220 g were paired according to the weight and then divided randomly into control and Shigella flexneri-treated groups. Shigella flexneri was administrated intragastrically in healthy male rats to induce bacillary dysentery. The effect of PAR-2 activation on the normal rectal motility was detected using tension recording system. The change of PAR-2-mediated effect was investigated at different time following the infection.

Results: The activation of PAR-2 induced a dose-dependent relaxation on the rectal longitudinal strips. This effect was TTX insensitive. After the infection with Shigella flexneri, the inhibitory effect induced by PAR-2 activation was impaired at day 11 and 18, then recovered to control level at day 25 and 35, besides, the basal mechanical activity of the rectal strips increased at day 11 following infection and recovered to control level at day 18, 25 and 35.

Conclusions: These data indicated that PAR-2 activation regulated the rectal motility and this effect was impaired following bacillary dysentery.

Keywords: IBS; PAR-2; Rectum; Bacillary Dysentery; Motility.

\section{Introduction}

In gastrointestinal tract, proteinase-activated receptor-2 (PAR2) can be activated by endogenous and exogenous proteinases, including mast cell and neutrophil derived tryptase and bacterial and parasite-derived proteinases to modulate inflammation, nociception, permeability, motility, and ion transport [1-5]. Numerous reports have also found that the motility disturbances has close relationship with inflammation, and the alteration and role of PAR-2 in gastrointestinal motility disturbances under intestinal infection or inflammation has also been investigated [610]. As we know, PAR-2 activation can modify the colon motility, including enhancement and attenuation [11, 12], it can induces relaxation of guinea pig internal anal sphincter [13], however, the regulatory effect of PAR-2 on the rat rectal motility and it's role following bacillary dysentery is uncertain. The aim of the present work was to investigate the role of PAR- 2 in the control of rectal motility and its change after intestinal infection. We hypothesized that PAR-2 inhibited the motility of rectum and this inhibitory effect was changed following the colitis induced by S. flexneri. For this purpose, normal and S. flexneri infected rats were used to study how rectal motility was affected by the PAR-2 activation under control conditions and in different post infectious phases. Spontaneous and PAR-2 mediated effect were assessed in vitro before and after the $\mathrm{S}$. flexneri infected by tension recording system.

\section{Material and Methods}

\section{Bacteria preparation}

Clinical isolates of S. flexneri were obtained from Clinical Laboratory, Qi Lu hospital, Ji Nan, China. The strains were routinely grown in blood plate at $37^{\circ} \mathrm{C}$, overnight.

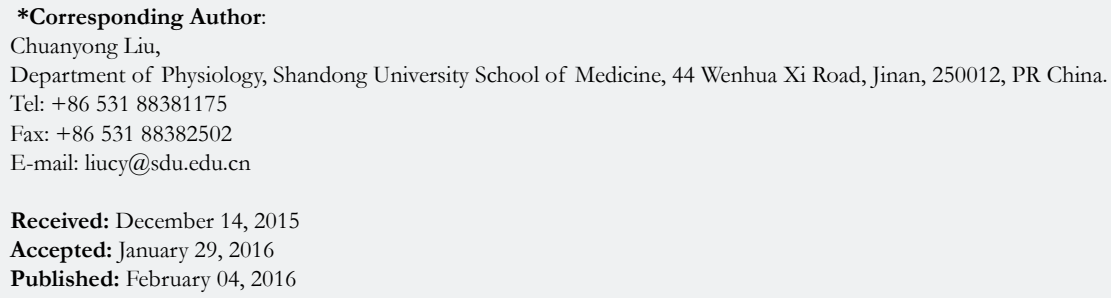

Copyright: Liu $\mathbf{C}^{\circ}$ 2016. This is an open-access article distributed under the terms of the Creative Commons Attribution License, which permits unrestricted use, distribution and reproduction in any medium, provided the original author and source are credited. 


\section{Intestinal infection and tissue preparation}

All experimental procedures were approved by the Committee on Animal Research and Ethics of Shandong University School of Medicine. The model was established according to our previous work [8]. Briefly, healthy male wistar rats, weighing 200-220 $\mathrm{g}$ (provided by Animal Center of Shandong University) were paired according to the weight and then divided randomly into control and S. flexneri-treated (SF-treated) groups. Rats received intragastric administration of inocula of $10^{8}$ colony forming units (CFU) in $1 \mathrm{ml}$ of sterile normal saline after an overnight fast (water ad libitum). The rats in the control group were treated with $1 \mathrm{ml}$ sterile normal saline. Rats were raised individually in cages and sacrificed by cervical dislocation on days 11, 18, 25 and 35 following the $\mathrm{S}$. flexneri treatment. Rectum $(0.5 \mathrm{~cm}$ from anus, 1-2 cm length) was excised and prepared for tension recording.

Recording of the tension of colonic muscle strips

A segment (1-2 cm length) of rectum was immediately removed from the control and SF-treated rats. The segment was opened along the mesenteric border and pinned flat in a dish filled with oxygenated Krebs solution. Full thickness muscle strips parallel to the long axis of the rectum ( $4 \mathrm{~mm}$ wide, $10 \mathrm{~mm}$ long) were prepared and then suspended in a organ bath which was filled with $5 \mathrm{ml} \mathrm{Krebs}$ solution, continuously perfused with $95 \% \mathrm{O}_{2}$ and $5 \% \mathrm{CO}_{2}$ and maintained at $37{ }^{\circ} \mathrm{C}$. The muscle mechanical activity was measured by an external isometric force transducer (JH-2B, Instrument Company of Chengdu, Chengdu, China) and a polygraph system (SMUP-PC, Fudan University, Shanghai, China). The muscle strips were required an equilibration for at least $45 \mathrm{~min}$ with flushing every $15 \mathrm{~min}$ under a preload of $1 \mathrm{~g}$. Then the mechanical response to the PAR-2-AP, SLIGRL-NH2, and PAR-2 reverse peptide (PAR-2-RP), LRGILS-NH2 were examined. In some experiments, muscle strips were pretreated with tetrodotoxin (TTX, $10 \mu \mathrm{M}$ ) for $30 \mathrm{~min}$ before administration of PAR-2-AP or PAR-2-RP.

\section{Chemicals}

PAR-2-AP (SLIGRL-NH2) and PAR-2-RP (LRGILS-NH2) were purchased from CL BIO-SCIENTIFIC CO., LTD (Xi an, China). TTX were purchased from Sigma-Aldrich Corp (St Louis, MO, USA).
The Krebs solution was composed of the following reagents (mM):

$\mathrm{NaCl}$ 120.6, $\mathrm{KCl}$ 5.9, $\mathrm{CaCl}_{2}$ 2.5, $\mathrm{KH}_{2} \mathrm{PO}_{4} 1.2, \mathrm{MgCl}_{2} 1.2, \mathrm{NaHCO}_{3}$ 15.4 and glucose 11.5 .

\section{Statistical analysis}

Data are expressed as mean \pm SEM. Comparisons between two groups were performed by Student's $t$-test, and comparisons among multiple groups were performed by one-way analysis of variance (ANOVA). $\mathrm{p}<0.05$ was considered as statistically significant. The mean value of the average tension for $3 \mathrm{~min}$ period before treatment with PAR-2-AP or PAR-2-RP was taken as the baseline. $R$ value $=$ the average tension for 2, 4, 6, 8, $10 \mathrm{~min}$ after each treatment/baseline. For TTX preincubation, baseline was the average tension for $3 \mathrm{~min}$ period before the application of PAR-2-AP or PAR-2-RP.

\section{Results}

Effect of PAR-2-AP on the spontaneous contraction of longitudinal muscle strips of rectum

PAR-2-AP was shown to inhibit the spontaneous contraction of rectal longitudinal muscle strips, but normal saline (NS) and PAR2-RP had no apparent effect on the mechanical activity of strips (Figure 1A). Exogenous PAR-2-AP (1-10 $\mu \mathrm{M})$ dose dependently inhibited the spontaneous contraction of longitudinal muscle strips of rectum at 2 min following PAR-2-AP $(5 \mu \mathrm{M}, 10 \mu \mathrm{M})$ administration (Figure 1B). The contraction of the rectal muscle strips decreased by $0.176 \pm 0.04(\mathrm{p}<0.05$ vs $\mathrm{RP}, \mathrm{n}=7)$ and 0.213 $\pm 0.04(\mathrm{p}<0.01$ vs $\mathrm{RP}, \mathrm{n}=7$ ) respectively.

\section{Effect of TTX on relaxation evoked by PAR-2-AP on rectal muscle strips}

In order to investigate the role of enteric nervous system (ENS) in the relaxation induced by PAR-2-AP, PAR-2-AP and PAR-2-RP were administrated $30 \mathrm{~min}$ following the pretreatment of TTX (10 $\mu \mathrm{M})$, the specific blocker of voltage dependent sodium channels on the membrane of nerve fibers. It seemed that pretreatment of TTX did not influence the inhibitory effect of PAR-2-AP on the contraction of rectal muscle strips (Figure 2).

Figure 1. The inhibition of PAR-2-AP on the spontaneous contraction of rectal muscle strip of rat. The representative recordings of the contraction of rectal muscle strips were shown in A, PAR-2-AP or RP was $10 \mu \mathrm{M}$. The dose-response was shown in B. $*_{p}<0.01, \# p<0.05$ vs RP.
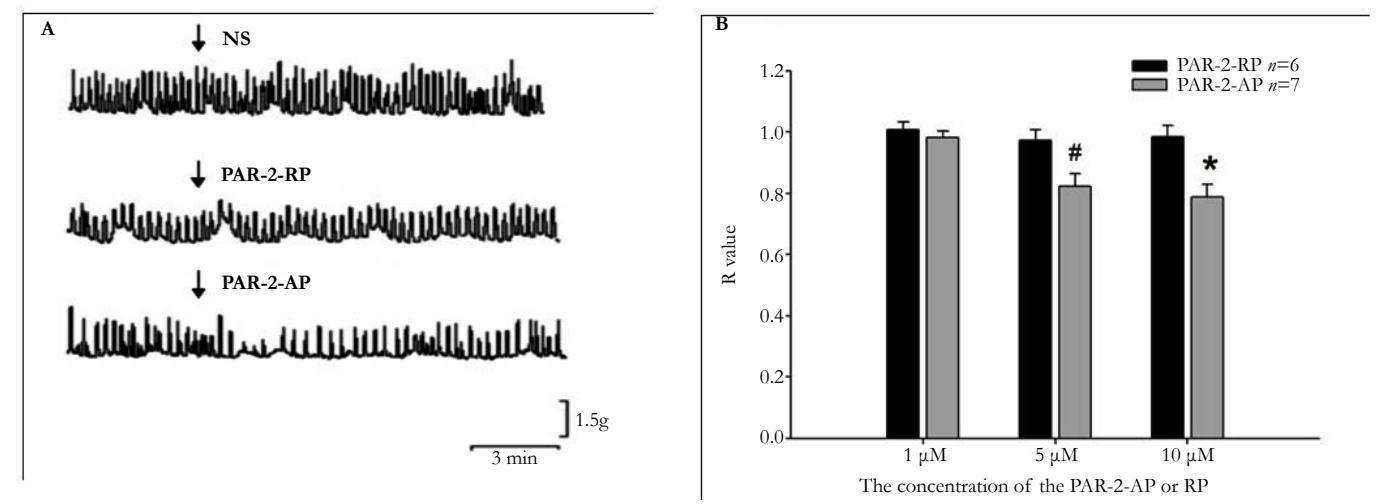


\section{Changes in PAR-2-AP induced relaxation following intestinal infection}

The $1 \mu \mathrm{M}$ PAR-2-AP had no effect on the contraction of rectal muscle strips in both control and SF-treated group (Figure 3), but the inhibition induced by $5 \mu \mathrm{M}$ PAR-2-AP significantly decreased in SF-treated group at 11 and 18 days, and this decrease recovered to control level at 25 and 35 days (Figure 3). The impaired relaxation was only observed at 11 days after the administration of $10 \mu \mathrm{M}$ PAR-2-AP in SF-treated rats (Figure 3).

Comparison of time course of $5 \mu \mathrm{M}$ PAR-2-AP induced relaxation on the rectal muscle strip prepared from control and SF-treated rats

In control group, the $5 \mu \mathrm{M}$ PAR-2-AP inhibited the mechanical activity of rectal muscle strips at day 11,18 and 25 , the effective time was shorten accompanied the time (Figure 4 A, B, C). In the infected group, the $5 \mu \mathrm{M}$ PAR-2-AP impaired the spontaneous contraction of rectal muscle strips only on day 25 (Figure $4 \mathrm{~A}$, $\mathrm{B}, \mathrm{C})$, there was no inhibitory effect on day 11 and 18. On day 35, $5 \mu \mathrm{M}$ PAR-2-AP had no effect on rectal muscle strip in both control and SF-treated groups (Figure 4D).

The spontaneous contractility of rectal muscle strips in both control and infectious groups

The spontaneous activity of the rectal muscle strips increased on day 11 post infectious compared to that of the control group. There was no significant difference between control and SFtreated groups on day 18, 25 and 35 (Figure 5).

\section{Discussion}

In this study, we described the inhibitory effect of PAR-2 activation on the rectal motility in vitro, we found changes in PAR2 function in rats following bacillary dysentery. Results obtained showed the impairment in PAR-2 dependent relaxation of rat rectal longitudinal muscle strips on day 11 and 18 postinfection, and this alteration recovered to control level on day 25 and 35 post infection. The basal spontaneous activity of the rectal muscle strips increased on day 11, then returned to control level.

As we know, the function of gastrointestinal tract is affected by the inflammation or infection, which contributes to the development of functional gastrointestinal disease, such as post infectious IBS. Rectum is vital in defecation, and the alterations in the pattern of defecation is the most common symptom in patients with IBS. It's reported that the compliance, tone and sensation of rectum were changed in patients with IBS [14], and the alterations in rectal sensitivity and motility were obersved in children with IBS [15]. Because approximately $1 / 10$ of IBS cases describe a post infectious onset and bacillary dysentery is a causative factor in PI-IBS [16, 17], so it's necessary to investigate the alteration of rectum motility following SF-infection. It's known that PAR-2 has a close relationship with IBS [18], so in our study, we examined the effect of PAR-2 on the motility of rectal muscle. Results showed a dose-dependent relaxation induced by PAR-2 activation. This effect was independent with enteric nervous system indicating the functional expression of PAR-2 on rectal smooth muscle cell. The changed expression and function of PAR-2 was reported in colon in chemical colitis models [9]. In Trichinella spiralis-infected (14 and 30 days postinfection) rats, the function and expression of PAR-2 on jejunum were also observed. The PAR-2 induced relaxation of jejunum was enhanced on day 14 postinfection (early phase),and motor responses were normalized on day 30 (early phase) [10]. Here, we also found that the inhibitory effect of PAR-2 on the motility of rectal muscle strip changed at different time post infection. The relaxation induced by PAR-2 activation was impaired on day 11 and 18 postinfection, and according to our previous study [8], there was the early postinfectious phase (11 and 18 days), then the impairment recovered to control level by day 25 and 35 (late phase). Meanwhile, the spontaneous contraction of the rectal muscle strips was tend to increase on day 11 (Figure 5) indicating that the changes in motor of PAR-2 activation associated with the early postinfectious state might have a beneficial role to promote the bacteria expulsion. Interestingly, by day 25 and 35 postinfection, both basal motility and diastolic responses to SLIGRL-NH2 were normalized. Therefore, changes in PAR-2-mediated motor responses were observed in association with altered basal motility and would contribute to normalize the rectum motility [10]. The change of the PAR-2 expression might be another reason for the impairment of PAR-2 induced relaxation. And several endogenous and exogenous proteinases are candidates to modulate postinfectious changes in PAR-2 expression and functionality. A few studies suggest that PAR-2 dysfunction is originated by a loss of receptors [8-10, 19]. This loss could be related to enhanced levels of proteinases including

Figure 2. The effect of TTX on the inhibitory effect induced by PAR-2-AP. The muscle strips were incubated with TTX $(10 \mu \mathrm{M}) 30$ min before the treatment of different concentration of PAR-2-AP or RP.

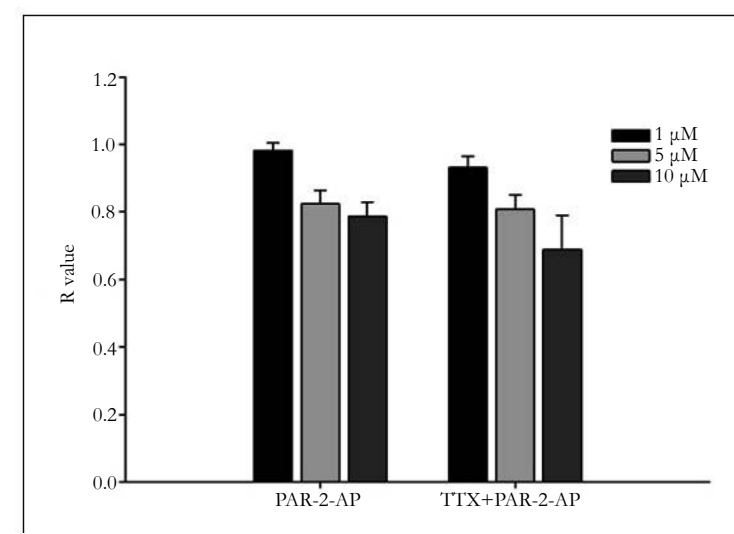


Figure 3. Comparison of the inhibitory effect of different concentration of PAR-2-AP on rectal muscle strips separated from control and SF-treated rats at different time postinfection. $\# \mathrm{p}<0.05$ vs control.

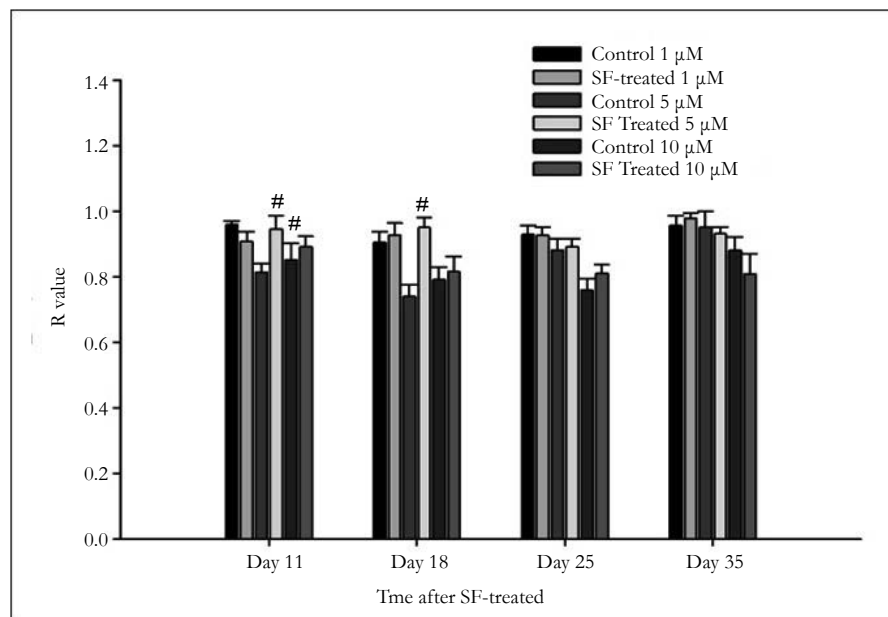

Figure 4. Time course of the inhibitory effect of $5 \mu \mathrm{M}$ PAR-2-AP on spontaneous contraction of the rectal muscle strip prepared from control and SF-treated rats at days 11(A), 18(B), 25(C), 35(D) postinfection. \#P<0.05 vs. normalized baseline, $*_{\mathrm{p}}<0.05$ vs control.
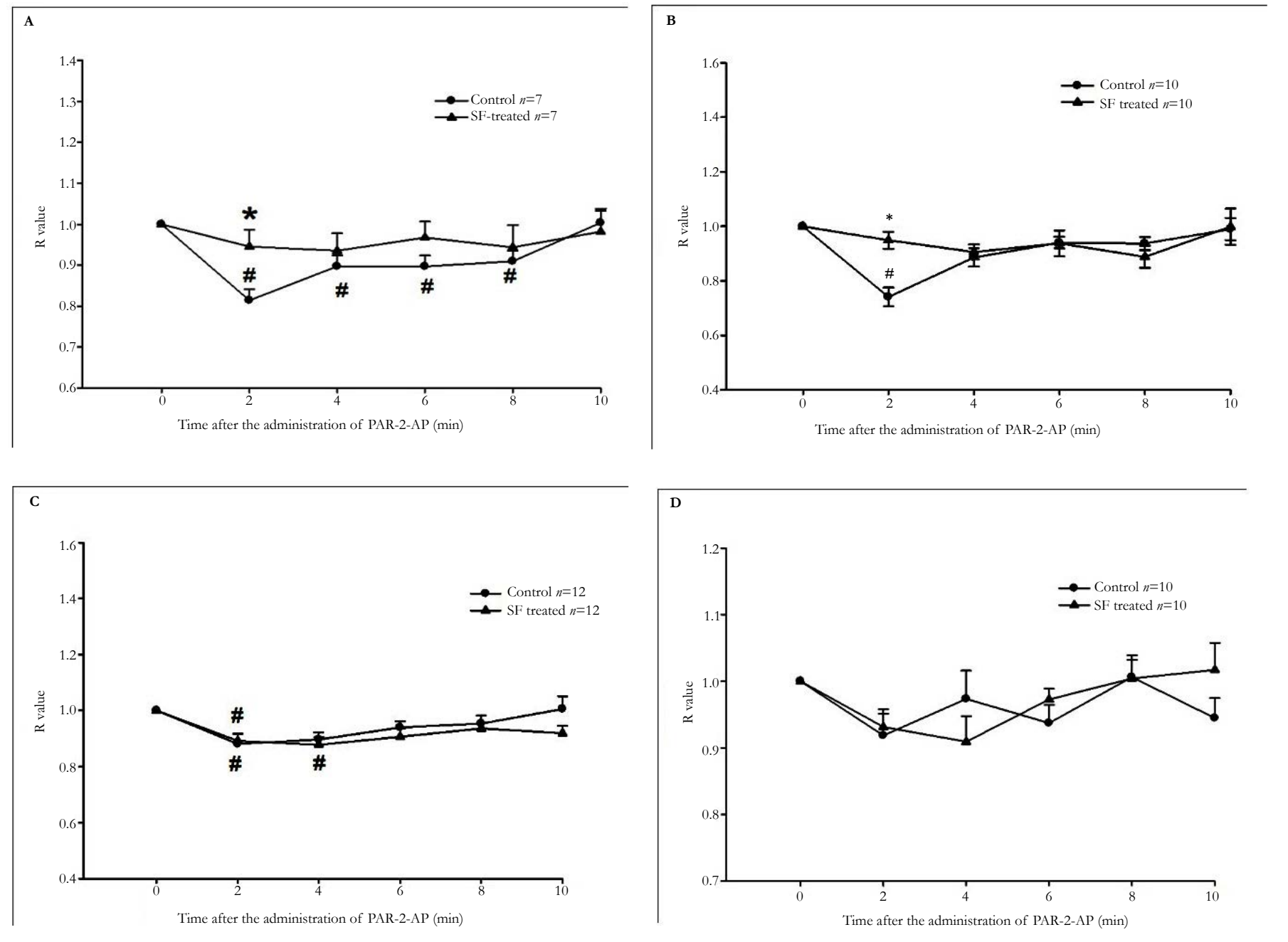

those produced by the bacteria, which would increase PAR2 activation and internalization leading to a reduced receptor presence on the cell surface [20]. In this respect, the infiltration of neutrophils after the infection of S. flexneri is one source of endogenous proteinases. Besides, the expression of PAR-2 was regulated by inflammatory cytokines in rat astrocytes and colon $[21,22]$ indicated a possible regulatory mechanism existing in the

model of S. flexneri infected rat for the release of LPS and other cytokines by bacteria and immune cells, which might contribute to the dysfunction of PAR-2 in our study. Actually in our previous work, the PAR-2 expression was down regulated in distal colon after the infection of S. flexneri [8].

Taken together, the contraction of rectal longitudinal strips was 
Figure 5. The spontaneous contractility in control conditions and at different postinfectious phases, expressed as the area under the cure (AUC) in 3 min. Data are means \pm SEM of $6-10$ rectal muscle. $\# \mathrm{p}<0.05$ vs control.

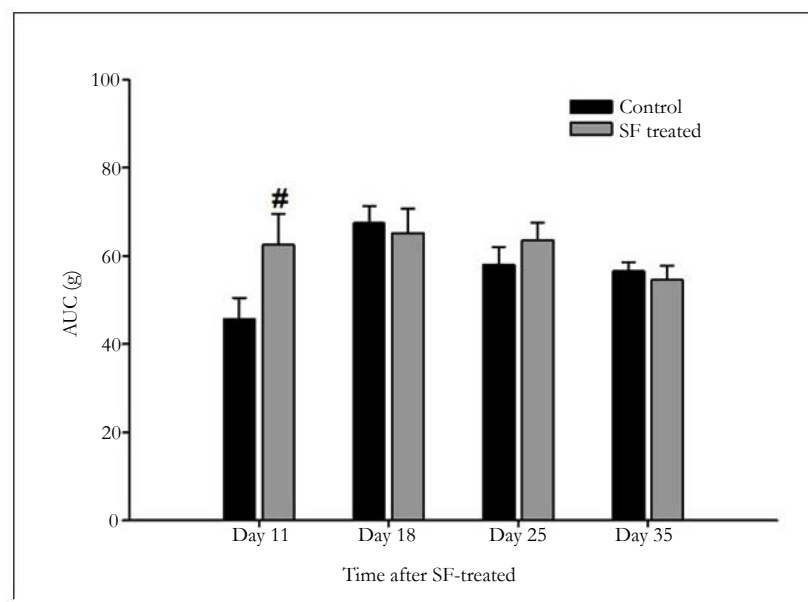

inhibited by the activation of PAR-2, this relaxation effect was impaired on day 11 and 18 postinfectious, the basal contraction of the rectal strips increased on day 11, both the increased basal mechanical activity and the impaired PAR-2-AP induced relaxation returned to normal level on day 25 and 35 postinfectious. These findings are helpful to understand the role of PAR-2 in the development of rectum related disease under inflammation or infection.

\section{Acknowledgements}

This work is supported by the Ningxia Natural Science Foundation No. NZ11121.

\section{References}

[1]. Adams MN, Ramachandran R, Yau MK, Suen JY, Fairlie DP, et al. (2011) Structure, function and pathophysiology of protease activated receptors. Pharmacol Ther 130(3): 248-282.

[2]. Hansen KK, Sherman PM, Cellars L, Andrade-Gordon P, Pan Z, et al. (2005) A major role for proteolytic activity and proteinase-activated receptor-2 in the pathogenesis of infectious colitis. Proc Natl Acad Sci USA 102(23): 8363-8368.

[3]. van der Merwe JQ, Hollenberg MD, MacNaughton WK (2008) EGF receptor transactivation and MAP kinase mediate proteinase-activated receptor-2-induced chloride secretion in intestinal epithelial cells. Am J Physiol 294(2): G441-G451

[4]. Coelho AM, Vergnolle N, Guiard B, Fioramonti J, Bueno L (2002) Proteinases and proteinase-activated receptor 2: a possible role to promote visceral hyperalgesia in rats. Gastroenterology 122(4): 1035-1047.

[5]. Kawabata A, Kuroda R, Nagata N, Kawao N, Masuko T, et al. (2001) In vivo evidence that protease-activated receptors 1 and 2 modulate gastrointestinal transit in the mouse. Br J Pharmacol 133(8): 1213-1218.

[6]. Kerckhoffs AP, Ter Linde JJ, Akkermans LM, Samsom M (2008) Trypsinogen IV, serotonin transporter transcript levels and serotonin content are increased in small intestine of irritable bowel syndrome patients. Neurogastroenterol Motil 20(8): 900-907.

[7]. Tahara T, Shibata T, Nakamura M, Yamashita H, Yoshioka D, et al. (2009) Promoter methylation of protease-activated receptor (PAR2) is associated with severe clinical phenotypes of ulcerative colitis (UC). Clin Exp Med 9(2): $125-130$
[8]. Luo Y, Wang F, Qin J, Feng M, Lv Y, et al. (2010) Long-term downregulation of protease-activated receptor-2 expression in distal colon in rats following bacillary dysentery. Regul Pept 163(1-3): 49-56.

[9]. Sato K, Ninomiya H, Ohkura S, Ozaki H, Nasu T (2006) Impairment of PAR-2-mediated relaxation system in colonic smooth muscle after intestinal inflammation. Br J Pharmacol 148(2): 200-207.

[10]. Fernández-Blanco JA, Hollenberg MD, Martínez V, Vergara P (2013) PAR2-mediated control of barrier function and motility differs between early and late phases of postinfectious gut dysfunction in the rat. Am J Physiol Gastrointest Liver Physiol 304(4): G390-400.

[11]. Suckow SK, Anderson EM, Caudle RM (2012) Lesioning of TRPV1 expressing primary afferent neurons prevents PAR-2 induced motility, but not mechanical hypersensitivity in the rat colon. Neurogastroenterol Motil 24(3): e125-135.

[12]. Mulè F, Baffi MC, Capparelli A, Pizzuti R (2003) Involvement of nitric oxide and tachykinins in the effects induced by protease-activated receptors in rat colon longitudinal muscle. Br J Pharmacol 139(3): 598-604.

[13]. Huang SC (2014) Proteinase-activated receptor-1 (PAR1) and PAR2 mediate relaxation of guinea pig internal anal sphincter. Regul Pept 189: 46-50.

[14]. Törnblom H, Van Oudenhove L, Tack J, Simrén M (2014) Interaction between preprandial and postprandial rectal sensory and motor abnormalities in IBS. Gut 63(9): 1441-1449.

[15]. Van Ginkel R, Voskuijl WP, Benninga MA, Taminiau JA, Boeckxstaens GE (2001) Alterations in rectal sensitivity and motility in childhood irritable bowel syndrome. Gastroenterology 120(1): 31-38.

[16]. Spiller RC (2004) Inflammation as a basis for functional GI disorders. Best Pract Res Clin Gastroenterol 18(4): 641-661.

[17]. Wang LH, Fang XC, Pan GZ (2004) Bacillary dysentery as a causative factor of irritable bowel syndrome and its pathogenesis. Gut 53(8): 1096-1101.

[18]. Roka R, Wittmann T, Bueno L (2008) Altered protease signalling in the gut: a novel pathophysiological factor in irritable bowel syndrome. Neurogastroenterol Motil 20(8): 853-856.

[19]. Shea-Donohue T, Notari L, Stiltz J, Sun R, Madden KB, et al. (2010) Role of enteric nerves in immune-mediated changes in protease-activated receptor 2 effects on gut function. Neurogastroenterol Motil 22(10): 1138-e291.

[20]. Devlin MG, Gasser RB, Cocks TM (2007) Initial support for the hypothesis that PAR2 is involved in the immune response to Nippostrongylus brasiliensis in mice. Parasitol Res 101(1): 105-109.

[21]. Sokolova E, Aleshin S, Reiser G (2012) Expression of protease-activated receptor (PAR)-2, but not other PARs, is regulated by inflammatory cytokines in rat astrocytes. Neurochem Int 60(3): 276-285.

[22]. Horie A, Nagai K, Ohkura S, Ohama T, Komatsu H, et al. (2009) Proinflammatory cytokines suppress the expression level of protease-activated receptor-2 through the induction of iNOS in rat colon. J Vet Med Sci 71(12): $1609-1615$. 